\title{
Continuous low-dose therapy with vinblastine and VEGF receptor-2 antibody induces sustained tumor regression without overt toxicity
}

\author{
Giannoula Klement, ${ }^{1}$ Sylvain Baruchel, ${ }^{2}$ Janusz Rak, ${ }^{1}$ Shan Man, ${ }^{1}$ Katherine Clark, ${ }^{1}$ \\ Daniel J. Hicklin, ${ }^{3}$ Peter Bohlen, ${ }^{3}$ and Robert S. Kerbel ${ }^{1}$
}

\begin{abstract}
${ }^{1}$ Sunnybrook and Women's College Health Sciences Centre, Biological Sciences Program, Division of Cancer Biology Research, and Toronto-Sunnybrook Regional Cancer Centre, Toronto, Ontario M4N 3M5, Canada; Department of Medical Biophysics, University of Toronto, Ontario, Canada

${ }^{2}$ Hospital for Sick Children, Department of Pediatrics, Division of Hematology/Oncology,

New Agent and Innovative Therapy Program, Toronto, Ontario M5G 1X8, Canada

${ }^{3}$ ImClone Systems Inc., New York, New York 10014, USA
\end{abstract}

Address correspondence to: Robert S. Kerbel, Sunnybrook and Women's College Health Sciences Centre, Biological Sciences, Division of Cancer Biology Research, Room S-218, 2075 Bayview Avenue, Toronto, Ontario M4N 3M5, Canada.

Phone: (416) 480-5711; Fax: (416) 480-5703; E-mail: kerbel@srcl.sunnybrook.utoronto.ca.

Received for publication November 2, 1999, and accepted in revised form February 25, 2000.

\begin{abstract}
Various conventional chemotherapeutic drugs can block angiogenesis or even kill activated, dividing endothelial cells. Such effects may contribute to the antitumor efficacy of chemotherapy in vivo and may delay or prevent the acquisition of drug-resistance by cancer cells. We have implemented a treatment regimen that augments the potential antivascular effects of chemotherapy, that is devoid of obvious toxic side effects, and that obstructs the development of drug resistance by tumor cells. Xenografts of 2 independent neuroblastoma cell lines were subjected to either continuous treatment with low doses of vinblastine, a monoclonal neutralizing antibody (DC101) targeting the flk-1/KDR (type 2) receptor for VEGF, or both agents together. The rationale for this combination was that any antivascular effects of the low-dose chemotherapy would be selectively enhanced in cells of newly formed vessels when survival signals mediated by VEGF are blocked. Both DC101 and low-dose vinblastine treatment individually resulted in significant but transient xenograft regression, diminished tumor vascularity, and direct inhibition of angiogenesis. Remarkably, the combination therapy resulted in full and sustained regressions of large established tumors, without an ensuing increase in host toxicity or any signs of acquired drug resistance during the course of treatment, which lasted for $>6$ months.
\end{abstract}

This article may have been published online in advance of the print edition. The date of publication is available from the JCI website, http://www.jci.org. J. Clin. Invest. 105:R1-R8 (2000).

\section{Introduction}

The current interest in developing antiangiogenic drugs to treat cancer-a concept first proposed by Folkman (1) - can be traced to a number of factors (2). One is the molecular elucidation of a number of angiogenic growth factors, such as VEGF, the angiopoietins, and the receptor tyrosine kinases expressed by activated endothelial cells of newly formed vessels for such growth factors (3). Such discoveries have provided a number of targets for the rational development of antiangiogenic drugs such as humanized anti-VEGF neutralizing antibodies (4) and agents that block receptors for VEGF or the angiopoietins (2,5-8). Another factor is that some antiangiogenic drugs may delay or even circumvent the problem of acquired drug resistance $(9,10)$ because they target the genetically stable endothelial cells of newly formed tumor blood vessels rather than genetically unstable tumor cells that are prone to mutate and develop resistance $(11,12)$.
In addition to such rationally designed antiangiogenic drugs, there is a surprisingly diverse and extensive list of angiogenesis inhibitors that were not originally designed to function as such, e.g., thalidomide, IFN- $\alpha$, and IL-12 (2). Intriguingly, this list may include many different conventional cytotoxic chemotherapeutic drugs (2), radiation (13), and hormonal ablation therapies $(14,15)$. With respect to chemotherapy, the presence of dividing endothelial cells in newly forming tumor blood vessels (16-18) should render such vessels-in contrast to their mature, quiescent counterparts found in normal adult tissues-sensitive to the cytotoxic effects of such drugs in a manner similar to dividing bone marrow, hair follicle, or gut mucosal cells (11). This hypothesized "collateral damage" to the tumor vasculature could conceivably contribute to the antitumor efficacy of chemotherapy in vivo (11). If so, it should follow, as we first proposed in 1991 (11), that even tumors consisting of tumor cells resistant to a par- 
ticular drug might still respond to that drug through such antivasculature "side effects." However, this presumably occurs infrequently, or at least not to any therapeutically meaningful extent, as most human cancers are intrinsically resistant to chemotherapy, or initially respond, only to recur as a result of overgrowth of drugresistant subpopulations. This is all the more perplexing given recent reports from several groups showing that a variety of conventional chemotherapeutic drugs can bring about significant antiangiogenic or antivascular effects in vivo in a number of assays of angiogenesis. These drugs include tubulin-inhibiting taxanes, camptothecin analogues, antimetabolites, anthracyclines, and platinum drugs (19-25).

Recent results from Folkman's laboratory have helped shed some light on this paradox (26). Chemotherapy is normally given acutely, usually in the form of bolus infusions at maximum tolerated doses (MTDs) with long rest periods (e.g., 3 weeks) between successive drug exposures. It was suggested that these rest periods provide the endothelial cell compartment of a tumor an opportunity to repair some of the damage inflicted by the chemotherapy (26). Browder et al. proposed that this repair process could be partially compromised by administering lower doses of a chemotherapeutic drug, such as cyclophosphamide, more frequently, e.g., weekly. In this regard, it is interesting to note that continuous low-dose administration of methotrexate for the treatment of arthritis may have an antiangiogenic basis (27). This hypothetical "antiangiogenic scheduling" of chemotherapy (26) optimizes antitumor/vascular side effects so that even a subline of the Lewis lung carcinomas previously selected in vivo for acquired resistance to the MTD of cyclophosphamide can be rendered sensitive again to the drug in vivo by using continuous low-dose therapy of the same drug (26).

We decided to test the effects of low-dose continuous chemotherapy as a possible antiangiogenic strategy with 1 major additional modification, namely, combination with an agent that blocks the function of VEGF receptor2 (flk-1/KDR) and, hence, VEGF itself. The rationale for testing this particular combination is based on the finding that a major function of VEGF is now recognized to be promotion of survival of endothelial cells comprising newly formed vessels $(15,28,29)$. Hence, the ability of such cells to cope with the damage inflicted by continuous low-dose exposure to a chemotherapeutic drug could be selectively and significantly impaired, given the highly restricted pattern of expression of VEGF receptors to activated endothelial cells $(3,6,7)$. This could reduce host toxicity and thereby allow for longer-term administration of the chemotherapeutic agent without sacrificing, and perhaps even improving, antitumor efficacy.

To test the rationale of this combination strategy reported here, we used vinblastine, a monoclonal anti-flk-1 neutralizing antibody called DC101 $(5,6)$, and poor prognosis-related human neuroblastoma cell lines grown as xenografts in SCID mice. Poor prognosis neu- roblastoma in children is usually treated with aggressive combination chemotherapy at MTDs, with or without a bone marrow transplant (30). However, such therapies are associated with severe side effects and are seldom effective. The purpose of these experiments was to develop a more acceptable alternative treatment and one that could also be applied in principle to other types of cancer.

\section{Methods}

Cells and culture conditions. Neuroblastoma cell lines SKN-MC and SK-N-AS were obtained from American Type Culture Collection (ATCC; Rockville, Maryland, USA) and expanded as a monolayer culture by serial passage on tissue culture plates (Nunc A/S, Roskilde, Denmark) in DMEM, 5\% FBS (GIBCO BRL, Grand Island, New York, USA). Human umbilical vein endothelial cells (HUVECs; Clonetics, San Diego, California, USA) were expanded on $1 \%$ gelatin-coated tissue culture plates in MCDB 131 culture medium (JRH Biosciences, Lenexa, Kansas, USA) supplemented with 5 $\mathrm{ng} / \mathrm{mL}$ basic fibroblast growth factor (bFGF) (R\&D Systems, Inc., Minneapolis, Minnesota, USA), 10 $\mu \mathrm{M} / \mathrm{mL}$ heparin (Wyeth-Ayerst, St. Laurent, Quebec, Canada), 10 ng/mL EGF (Upstate Biotechnology Inc., Lake Placid, New York, USA), and 10\% FBS.

In vitro determination of drug sensitivity. Three thousand cells in $200 \mu \mathrm{L}$ growth media per well were plated in 96well flat-bottom tissue culture plates (Nunc A/S) and incubated at $37^{\circ} \mathrm{C}, 5 \% \mathrm{CO}_{2}$ for 24 hours before initiation of treatment. The cells were then washed with PBS and treated with 1-500 $\mathrm{ng} / \mathrm{mL}$ vinblastine sulfate (Calbiochem/Novabiochem Corp., San Diego, California, USA) for 24 hours, in groups of 8 wells per dose. The cells were then pulsed for 6 hours with $2 \mu \mathrm{Ci}$ /well of methyl- $\left[{ }^{3} \mathrm{H}\right]$ thymidine (Amersham Life Science, Buckinghamshire, United Kingdom). The plates were frozen and thawed, and the DNA was harvested onto a filtermat using a Titertek Cell Harvester (Flow Laboratories Ltd., Irvine, Ayr, UK). Incorporated radioactivity was measured on Wallac 1205 BetaPlate Scintillation Counter (Wallac Oy, Turku, Finland), and proliferation was expressed as a percentage of $\left[{ }^{3} \mathrm{H}\right]$ thymidine in treated cells versus that in controls.

In vivo tumor growth assessment. SK-N-MC cells were harvested using 1\% trypsin EDTA (GIBCO BRL), and a single-cell suspension of $2 \times 10^{6}$ cells in $0.2 \mathrm{~mL}$ of growth medium was injected subcutaneously into the flanks of 4- to 6-week-old CB-17 SCID mice (Charles River, St. Constant, Quebec, Canada). Approximately 3 weeks later, most tumors had grown to approximately $0.75 \mathrm{~cm}^{3}$, and mice were randomized into groups of 5 animals. Two independent experiments were performed, each totaling 20 animals in 4 groups. The treatment was as follows: group I (control), $0.4 \mathrm{~mL}$ of PBS (DC101 vehicle) intraperitoneally every 3 days and $0.15 \mathrm{~mL}$ injectable saline (Vinblastine vehicle) intraperitoneally every 3 days; group II, $0.4 \mathrm{~mL}$ of $2 \mathrm{mg} / \mathrm{mL}$ DC101 antibody ( $800 \mu \mathrm{g} /$ mouse) (31) every 3 days and $0.15 \mathrm{~mL}$ of injectable saline intraperitoneally every 3 
days; group III, vinblastine sulfate $0.75 \mathrm{mg} / \mathrm{m}^{2}(\sim 0.25$ $\mathrm{mg} / \mathrm{kg})$ intraperitoneal bolus at the start of therapy, followed by $1 \mathrm{mg} / \mathrm{m}^{2}$ per day $(\sim 0.33 \mathrm{mg} / \mathrm{kg}$ per day) via subcutaneous Alzet osmotic pumps (Alza Corp, Palo Alto, California, USA) for 3 weeks, followed by maintenance therapy with $0.15 \mathrm{~mL}$ of $0.067 \mathrm{mg} / \mathrm{mL}$ vinblastine sulfate $\left(1.5 \mathrm{mg} / \mathrm{m}^{2}\right)$ intraperitoneally every 3 days, and $0.4 \mathrm{~mL}$ of PBS intraperitoneally every 3 days; and group IV, combination of DC101 and vinblastine at doses identical to the single-agent groups.

SK-N-AS cells were harvested using $1 \%$ trypsin EDTA, and a single-cell suspension of $2 \times 10^{6}$ cells in $0.2 \mathrm{~mL}$ of growth medium was injected subcutaneously into the flanks of 4- to 6-week-old CB-17 SCID mice. Approximately 3 weeks later (average tumor size, $0.25 \mathrm{~cm}^{3}$ ), the mice were randomized into groups of 5 animals and treated as follows: controls, $0.4 \mathrm{~mL}$ of PBS and $0.15 \mathrm{~mL}$ of saline twice weekly; DC101 group, $800 \mu \mathrm{g}$ of DC101 $\mathrm{mAb} /$ mouse and $0.15 \mathrm{~mL}$ of saline intraperitoneally twice weekly; vinblastine group, $0.4 \mathrm{~mL}$ of PBS and 1.5 $\mathrm{mg} / \mathrm{m}^{2}(\sim 0.5 \mathrm{mg} / \mathrm{kg})$ vinblastine twice weekly; and combination therapy group, treated with DC101 and vinblastine at doses identical to the single-agent group.

The body weight, tumor size, and general clinical status of the animals were recorded every 2-3 days. Perpendicular tumor diameters were measured using a Vernier scale caliper, and tumor volume was estimated using the formula for ellipsoid: (width ${ }^{2} \times$ length) $/ 2$. Growth curves were statistically analyzed using repeated measures ANOVA.

The mice were sacrificed when tumor size reached 1.5 $\mathrm{cm}^{3}$ or $7.5-10 \%$ of their body weight as required by institutional guidelines. All other animal care was in accordance with institutional guidelines as well.

Histology. All tumors were excised, fixed in 10\% (vol/vol) formalin, and processed for immunohistochemical analysis. To obtain adequate tissue for the combination treatment group, 2 mice were sacrificed at 7.5 weeks of treatment. For single-agent and control groups, the tissues were collected and analyzed at the conclusion of the treatment, i.e., when the mice had to be sacrificed. Paraffin blocks were cut to $5-\mu \mathrm{m}$ sections and stained with hematoxylin and eosin (H\&E) for morphology evaluation and with the Apoptosis Detection System (Promega Corp., Madison, Wisconsin, USA) for assessment of programmed cell death.

Relative tumor vascularity assessed by an FITC-dextran perfusion assay. This method was designed to assess the relative functionality of the tumor vasculature. A suspension of $2 \times 10^{6}$ SK-N-AS neuroblastoma cells in 0.2 $\mathrm{mL}$ of media was injected into the flanks of CB-17 SCID mice. Tumors were allowed to grow to approximately $0.25 \mathrm{~cm}^{3}$, at which point tumor bearing mice were then treated with $1 \mathrm{mg} / \mathrm{m}^{2}(\sim 0.33 \mathrm{mg} / \mathrm{kg})$ vinblastine intraperitoneally every 3 days, or $800 \mu \mathrm{g}$ DC101 intraperitoneally every 3 days, a combination of the 2 agents, or saline. At 14 days, when divergence in tumor growth between the treatment groups was clearly evident, $0.2 \mathrm{ml}$ of $25 \mathrm{mg} / \mathrm{mL}$ FITC-dextran in
PBS (Sigma Chemical Co., St. Louis, Missouri, USA; average molecular weight of $148 \mathrm{kDa}$ ) was injected systemically into the lateral tail vein of each mouse and allowed to circulate for 20-30 minutes. Mice were then sacrificed by cervical dislocation, and blood samples collected by cardiac puncture into heparinized tubes for assessment of systemic fluorescein levels. Tumors were resected from the surrounding connective tissue, being careful to avoid spillage of intravascular contents; weighed; and placed into tubes containing 1:10 dispase (Collaborative Research, Two Oaks, Bedford, Massachusetts, USA). To normalize for dilution caused by the difference in tumor sizes, $1 \mathrm{~mL}$ of 1:10 dispase was added per $0.5 \mathrm{~g}$ of tissue. Tumors were incubated in a dark $37^{\circ} \mathrm{C}$ shaker overnight. The tissue was homogenized, centrifuged at $3,000 \mathrm{~g}$ for 10 minutes, and supernatant was collected and stored in the dark until further analysis. Similarly, the blood samples were centrifuged immediately after collection, and plasma was separated and protected from light at $4^{\circ} \mathrm{C}$ until analysis. Fluorescence readings were obtained on a FL600 Fluorescence Plate Reader (Biotek Instruments Inc., Winooski, Vermont, USA) from a standard curve created by serial dilution of the FITCdextran used for injection. The ratio of tumor fluorescence/plasma fluorescence was assumed to be reflective of the degree of tumor perfusion.

In vivo angiogenesis assessment by the Matrigel plug assay. Matrigel (Collaborative Biomedical Products, Bedford, Massachusetts, USA; refs. 5 and 32) stored at $-20^{\circ} \mathrm{C}$, was thawed at $4^{\circ} \mathrm{C}$ overnight, and mixed with $500 \mathrm{ng} / \mathrm{mL}$ bFGF. A total of $0.5 \mathrm{~mL}$ of this mixture was then injected subcutaneously into the shaved flanks of twenty 6- to 8week-old female Balb/cJ mice (The Jackson Laboratory, Bar Harbor, Maine, USA). Five mice, used as negative controls, were injected with Matrigel alone. Three days later, treatment mice were randomized into 4 groups as follows: group I, saline intraperitoneally; group II, $800 \mu \mathrm{g}$ DC101 intraperitoneally; group III, $1 \mathrm{mg} / \mathrm{m}^{2}$ vinblastine intraperitoneally; and group IV, combination therapy. All 25 mice were treated on days 4 and 7 and sacrificed on day 10. On day $10,0.2 \mathrm{~mL}$ of $25 \mathrm{mg} / \mathrm{mL}$ FITC-dextran in PBS was injected systemically into the lateral tail vein of each mouse and allowed to circulate for 20-30 minutes. Mice were then sacrificed by cervical dislocation, blood samples collected by cardiac puncture into heparinized tubes, centrifuged immediately after their collection, and plasma was separated and protected from light at $4^{\circ} \mathrm{C}$. The Matrigel plugs were resected from surrounding connective tissues, placed into tubes containing $1 \mathrm{~mL}$ of $1: 10$ dispase, and incubated in the dark in a $37^{\circ} \mathrm{C}$ shaker overnight. The following day, the plugs were homogenized and centrifuged at 3,000 g for 10 minutes, and supernatant was saved in the dark for analysis of fluorescence. Fluorescence readings were obtained on FL600 Fluorescence Plate Reader using a standard curve created by serial dilution of FITC-dextran used for injection. Angiogenic response was expressed as a ratio of Matrigel plug fluorescence/plasma fluorescence. 


\section{Results}

In vitro determination of differential drug sensitivity. Before undertaking our in vivo experiments, we established a dose of vinblastine at which significant antiproliferative effects on endothelial cells, but not tumor cells, were observed. To do so, we optimized growth conditions to achieve comparable levels of proliferative activity in 2 human neuroblastoma cell lines (SK-NM-C and SK-N-AS) and HUVECs. All 3 cell lines were grown in DMEM with $10 \%$ FBS, but the HUVECs were grown on gelatinized plates and in the presence of additional growth factors (bFGF and EGF). The untreated controls show similar levels of $\left[{ }^{3} \mathrm{H}\right]$ thymidine incorporation for all 3 cell lines, thus eliminating the concern that the differences in proliferation may be inherent (Figure 1). At the higher concentrations of vinblastine used (e.g., 100-400 ng/mL), all 3 cell populations were strongly inhibited, especially HUVECs. In striking contrast, at the lowest concentrations (e.g., 0.78 $\mathrm{ng} / \mathrm{mL}$ ) vinblastine retained almost the same degree of inhibitory activity against HUVECs, whereas antiproliferative activity against 2 tumor cell lines was lost (Figure 1). The source of this striking differential sensitivity is not clear, but it should be noted that at least 1 of the tumor cell lines, SK-N-MC, is positive for multidrug resistance-associated protein (MRP), which can result in vinblastine resistance (33). These in vitro findings suggest that the lowering of the usual MTD used in the clinic may not compromise vinblastine activity against (dividing) endothelial cells present in tumor blood vessels.

In vivo tumor growth assessment. Building on this in vitro difference in sensitivity to vinblastine, we went on to evaluate the effects of lower doses of vinblastine in an in vivo model, using an increased dose frequency to maximize the potential for endothelial injury. Xenografts of either SK-N-MC neuroepithelioma or SK-N-AS neuroblastoma cell lines were implanted subcutaneously in the flanks of 4- to 6-week-old CB-17 SCID mice and grown to approximately $0.75 \mathrm{~cm}^{3}$ before initiation of treatment. The SK$\mathrm{N}-\mathrm{MC}$ cell line, which expresses MRP (33), was chosen for long-term experiment to assess for the development of drug resistance. In contrast, the SK-N-AS, a highly tumorigenic and vascular neuroblastoma cell line, which has not been studied, to our knowledge, for its chemoresistance, was chosen for the short-term experiment and tumor perfusion studies.
Table 1

Summary of neuroblastoma xenograft responses to antiangiogenic therapy

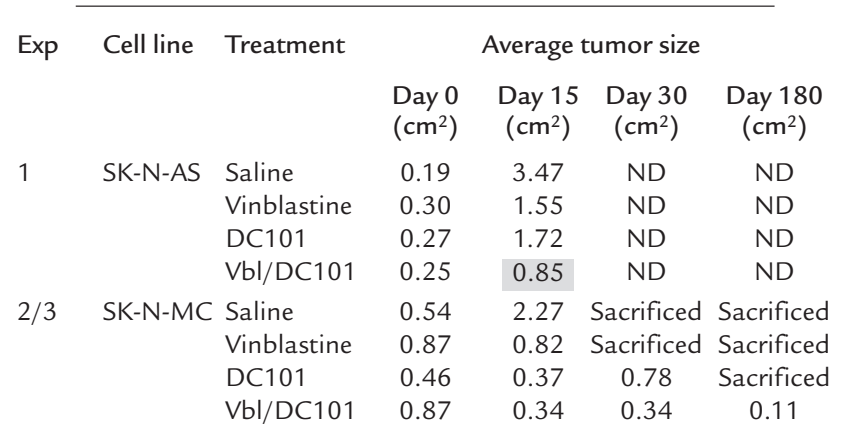

There is a marked initial response to both single agents such that in SK-N-MC xenografts, the response to DC101 at 15 days is almost equal to that of the combination-treatment group. The case of SK-N-AS shows the limitations of measuring tumor volume by external dimensions (Day 15, Vbl/DC101-shaded box). The ulcerated, scabbed tumor retains its external dimensions despite evidence of vascular compromise (see Fig. 5), ulceration and necrosis. Over time there is a delayed divergence effect such that neither of the single agents alone is able to result in sustained tumor regressions whereas combination treatment induces a lasting tumor regression. Sacrificed refers to animals which were euthanized because their tumors reached a size of $1.5 \mathrm{~cm}^{3}$ (or $10-15 \%$ of body weight), as required by our institutional guidelines. ND, time points not done.

The major findings in the long-term experiment, using SK-N-MC human neuroblastoma xenografts were as follows: (a) The first treatment group, treated with DC101, an anti-flk-1 receptor antibody shown previously to inhibit growth of different kinds of human xenografts in mice and in murine tumor models (5), showed the anticipated effectiveness in inhibiting tumor growth (Figure 2 , top panel, full circles; Table 1, day 15), but this effect was not sustained. (b) The findings in the second treatment group (vinblastine alone) were even more surprising. This agent, traditionally thought to act by inhibiting tumor cell proliferation through inhibition of tubulin assembly, even though used at "subclinical" low dose, produced significant, albeit not sustained, regression of tumor growth (Figure 2, top panel, filled squares; Table 1, day 15). (c) This growth delay in the vinblastine group was further potentiated with the simultaneous treatment of the anti-flk-1 antibody, DC101. The combination treatment induced an initial response comparable to the other treatment groups but then caused further, long-
Figure 1

Differential in vitro sensitivity to vinblastine of HUVECs and human neuroblastoma cells (SK-N$\mathrm{MC}$ and SK-N-AS). Proliferation rates of the 3 cell lines were assessed by measuring $\left[{ }^{3} \mathrm{H}\right]$ thymidine incorporation across a wide range of vinblastine concentrations. The most significant differences in sensitivity to vinblastine are evident at lower concentrations, whereas both neuroblastoma cell lines continued to incorporate thymidine at $80-90 \%$ of control rates, whereas HUVECs rates fell to $6.2 \%$ of control. Averages of 8 wells per dose and the corresponding SDs are shown.

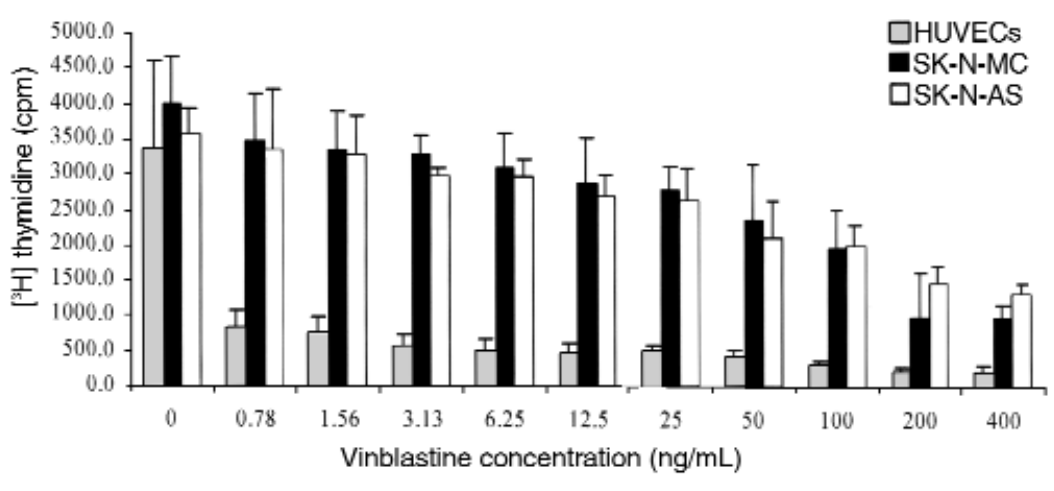




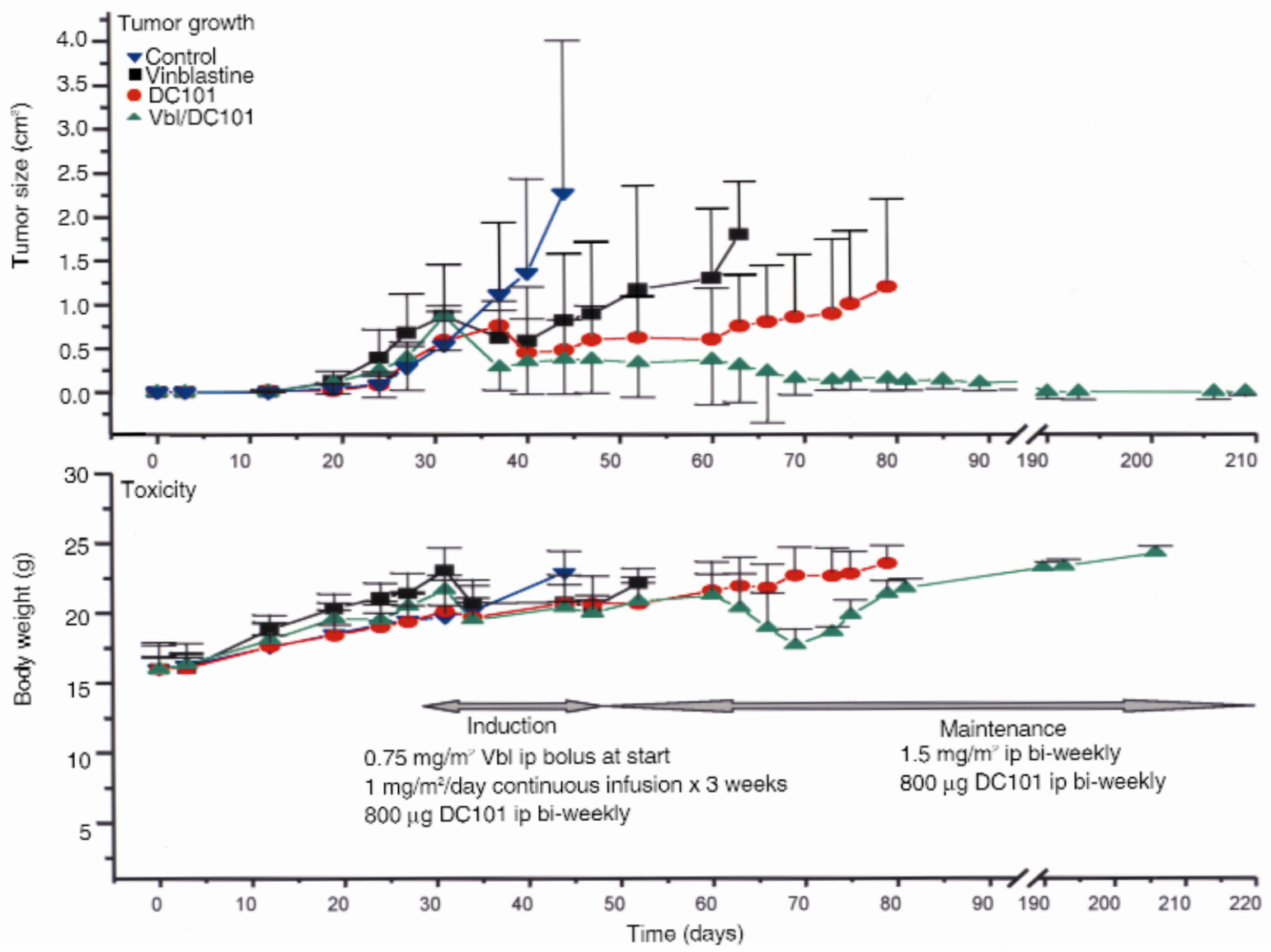

Figure 2

Induction of solid tumor regression by nontoxic, antiangiogenic combination therapy with low-dose vinblastine and anti-flk-1 antibody (DC101). Top panel: Established xenografts of human neuroblastoma (SK-N-MC) were treated by a putative antivascular regimen of low-dose vinblastine (induction: $0.75 \mathrm{mg} / \mathrm{m}^{2}$ bolus intraperitoneally; $1 \mathrm{mg} / \mathrm{m}^{2}$ per day continuous subcutaneous infusion for 3 weeks; maintenance: $1.5 \mathrm{mg} / \mathrm{m}^{2} \mathrm{every}$ 3 days) alone or in combination with an anti-VEGF-R2 antibody (DC101; $800 \mu \mathrm{g}$ every 3 days). There is an appreciable tumor growth inhibition by each of the single agents, which is comparable, at least initially, with that of the combination treatment group. The benefit of the combination treatment is most evident after prolonged treatment, when lasting and complete tumor regression is observed. The data are a compilation of 2 independent experiments, with the initial experiment lasting 34 days and the second still ongoing (> 210 days). In both sets, 20 mice were randomized into 4 groups. Bottom panel: Lack of toxicity-dependent weight loss in mice bearing SK-N-MC tumor xenografts treated with "antivascular" vinblastine regimen alone or in combination with and anti-VEGF-R2 (DC101) antibody. There are no significant differences in weight between the groups, except for a transient (14-18 days long) episode of weight loss associated with diarrhea in the combination treatment group. The episode resolved without interruption of therapy. Average body weights $(\mathrm{g}) \pm \mathrm{SD}$ are plotted $(n=3-10$ mice). ip, intraperitoneally.

term, tumor regressions (Figure 2, top panel, full upward triangles; Table 1, experiments 2 and 3). (d) To date, the mice in combination therapy group have not manifested any resistance to the treatment or recurrence of disease, despite almost 7 months of continuous treatment. The mice remain healthy, with almost no evidence of tumor, except for a small, barely palpable remnant in 1 of the mice. The tumor sizes between the control and treatment group were statistically significant $(P=0.05)$ at days $30-45$ and between the treatment groups at days 30-80.

The response in the SK-N-AS was even more rapid producing significant differences in tumor growth within 2 weeks of treatment (Table 1) without an induction regimen. The macroscopic changes in tumor vascularity (see Figure $5 \mathrm{~b}$ ) are supported by the findings in 1 tumor perfusion study (see later here).

Also of interest, the 3 remaining mice in the long-term experiment were taken off the long-term combined therapy at 210 days of treatment and did not show any evidence of tumor relapse/regrowth 3 months later (G. Klement et al., unpublished observations).

Toxicity evaluation. Antivascular therapy would be expected to show minimal toxicity in the postnatal stage of development. To evaluate this aspect of DC101/vinblastine combination therapy, the health status of the mice was monitored. Weight was plotted at regular intervals and considered a surrogate for evaluation of systemic well-being, anorexia, or failure to thrive. As shown in Figure 2 (bottom panel), no significant differences in weights were seen between the 4 groups. The weight curve of the DC101 group parallels very closely that of the control group. The vinblastine group showed some weight gain retardation, but the differences never became significantly different from controls, and the mice contin- 
ued to grow. Similarly, the toxicity profile in the combination treatment group was very similar to those in the single-agent groups, with the exception of a transient episode of weight loss associated with diarrhea. The episode lasted approximately $2-3$ weeks and was unlikely to be due to the therapy as the mice recovered without interruption of treatment.

Other usual signs of drug toxicity in mice, such as ruffled fur, anorexia, cachexia, skin tenting (due to dehydration), skin ulcerations, or toxic deaths (21), were not seen at the doses used in our experiments. Diarrhea, a common sign of vinblastine toxicity when doses of 10

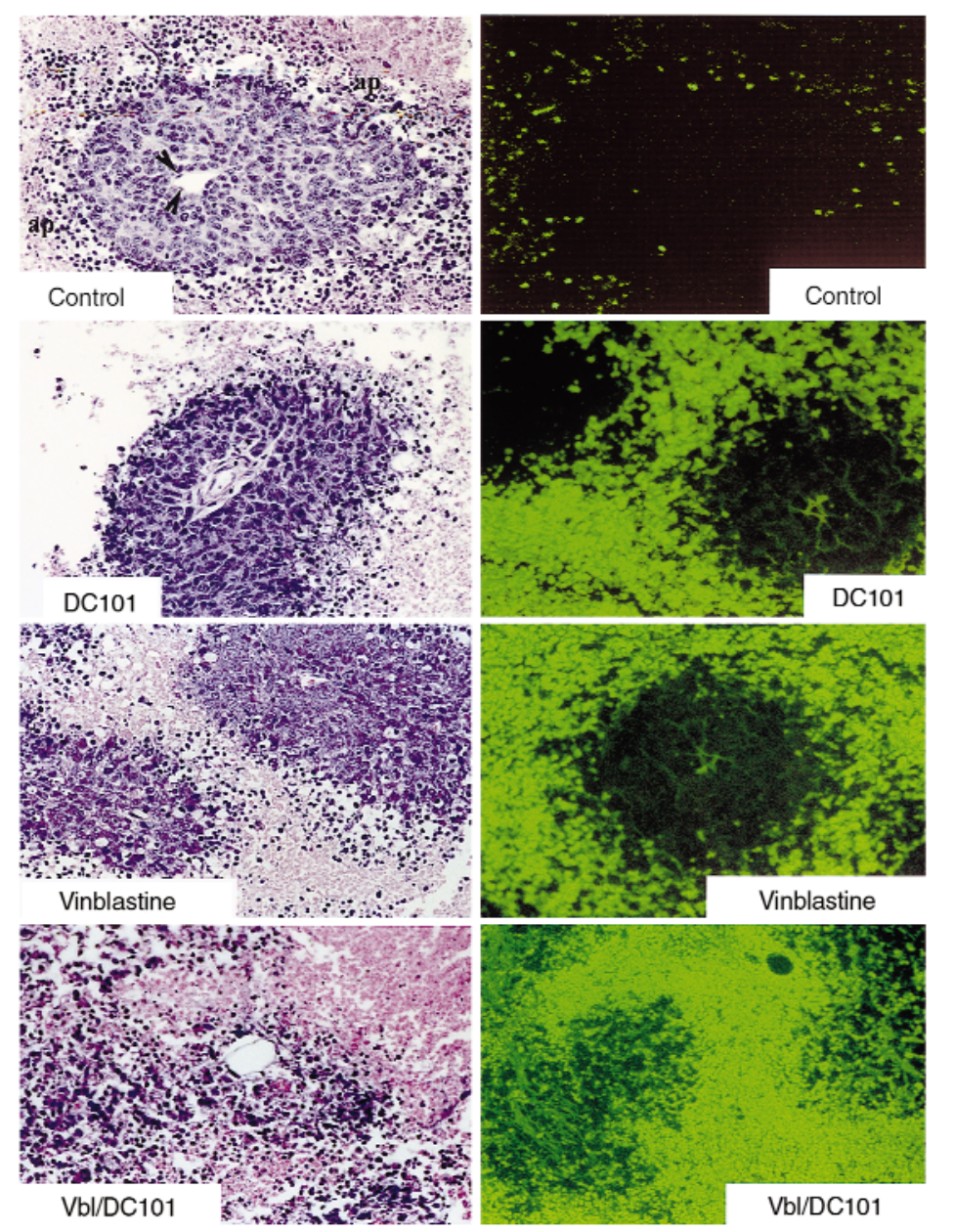

Figure 3

Vinblastine, DC101, or combination therapy induces tumor cell apoptosis in perivascular cuffs of SK-N-MC tumor xenografts. H\&E stain of formalin-fixed, paraffin-embedded sections. The typical tissue architecture (control, top two panels) shows perivascular cuffing by neoplastic cells and normal endothelial cell lining (arrows). Apoptotic cells (ap) are seen only at the periphery of the cuff, and their presence is confirmed by TUNEL staining (right-sided panels). In both single-treatment groups (vinblastine and DC101), widening of the apoptotic rims, and extension of the apoptotic figures into the cuff can be observed after 35 and 50 days of treatment, respectively. Viable malignant cells are still present within the tumor cuff in both single-agent groups. In contrast, histology of the combined therapy group reveals diffuse tumor cell death and total loss of preexisting architecture (bottom left-hand panel), a finding supported by the diffuse TUNEL stain in corresponding specimens (bottom right-hand panel). $\mathrm{mg} / \mathrm{kg}(21)$ are used, was generally not observed, except or the previously mentioned transient episode.

Histological analysis. To elucidate further the mechwith vinblastine, DC101, or the combined therapy, tissue histopathology assessment was undertaken. The typical tissue architecture of untreated SK-N-MC tumors is shown in Figure 3 (control panels). Cancer cells with high nuclear to cytoplasmic ratio form cuffs around central sels, and apoptotic cells characterized by pyknotic nuclei and cytoplasmic blebbing, are only evident as a thin rim at the periphery of the cuffs. The nuclei of these cells stain strongly for terminal deoxynucleotidyl transferase (TUNEL) reactivity, as expected for cells undergoing apoptosis. Vinblastine alone or DC101 treatment alone both show an increase in the width of the apoptotic rims (Figure 3), suggesting the cells most distal to the tumor vasculature are primarily affected, but a large percentage of viable tumor cells still survive in the center of the cuff. In both single-agent groups, specimens were collected at the time mice had to be sacrificed for ethical concerns regarding tumor burden. Despite the evident increase in apoptosis, there was a net tumor growth. In contrast, histology of the combined therapy group, as would be predicted by the regression in tumor size in this treatment group at the time of analysis (7.5 weeks of treatment), shows overwhelming loss of both cell viability and preexisting tumor architecture (Figure 3, bottom panels). There is a close similarity of the appearance of H\&E and TUNEL stain. Interestingly, we observed signs of endothelial cell toxicity in all of the treatment groups (Figure 4). Rather than a typical single layer of flattened endothelial cells surrounding the vascular lumen in untreated group (Figure 4a), we observed edema (Figure 4, b and c) and detachment from surrounding basement membrane (Figure 4, c-e) leading to complete vascular wall disintegration and tumor cell death (Figure 4f). Even though the actual examples depicted in Figure 4 are those in the DC101 group, their appearance is shared between the groups, and it is only the degree of tumor vessel regression that results in the seen differences in tumor size.

Tumor perfusion by assessment of intravascular fluorescence. To explore further the possibility that tumor regression induced with treatment using DC101 and/or vinblastine was indeed due to the vascular injury, rather than a direct antitumor cell effect, we assessed tumor perfusion directly by using a FITCdextran fluorescence method. Mice carrying established subcutaneous SK-N-AS human neuroblastoma xenografts $\left(\sim 0.25 \mathrm{~cm}^{3}\right)$ were 

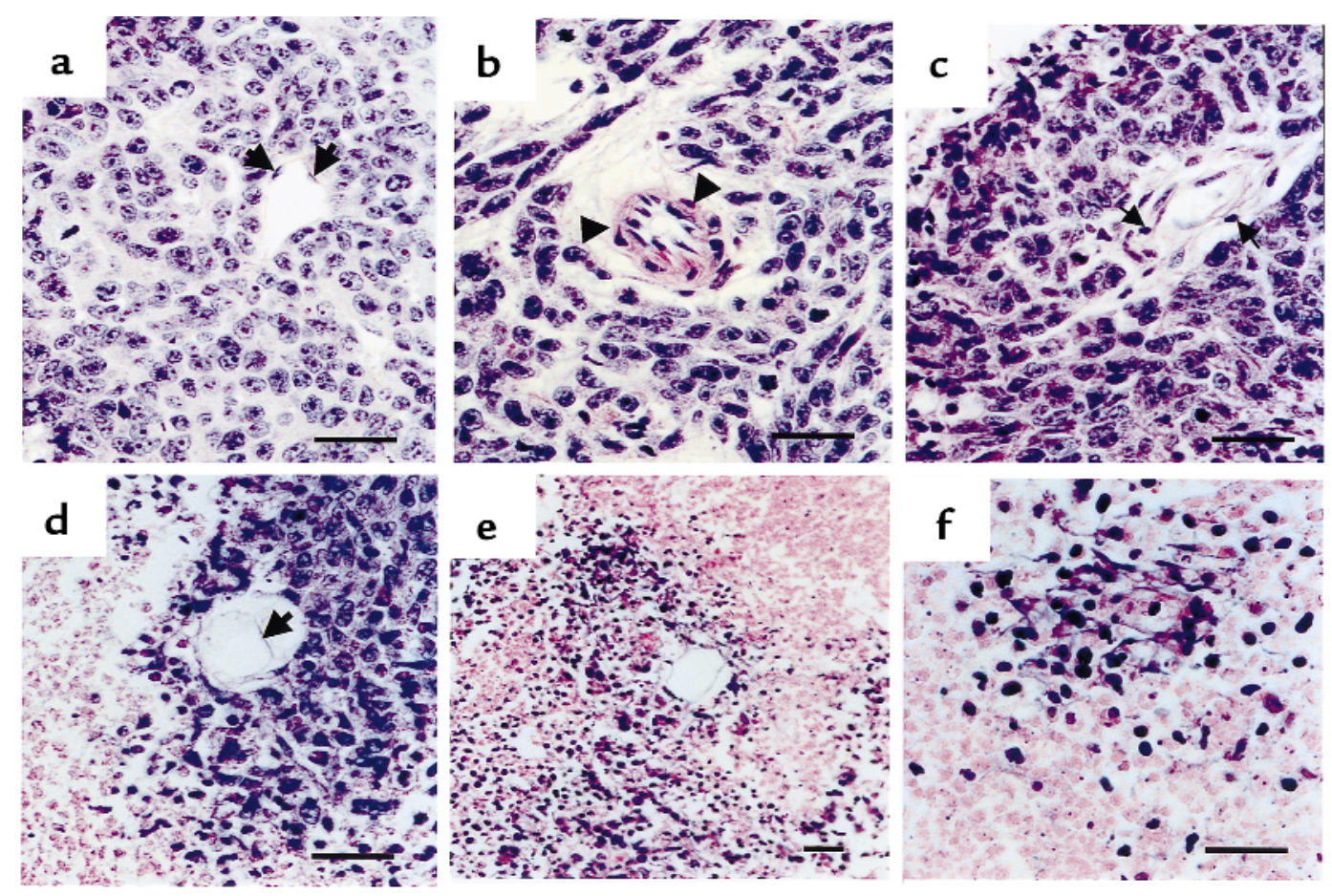

Figure 4

Morphological features of vascular damage induced by antiangiogenic therapy. H\&E stain of formalin-fixed, paraffin-embedded sections of the DC101 treatment group. These changes are common to all the treatment groups, but the prevalence and the severity of these changes were greatest in the combination treatment group. The typical slim single layer of endothelial cells, lining the vascular lumen in the untreated group (a, black arrows). Treatment with DC101 (35 days) and vinblastine (50 days) leads to various degrees of vascular wall disintegration. Edema and lymphocytic infiltration (arrows) are seen in both arterioles (b) and venules (c) of the tumor. Further injury resulted in detachment of endothelial cells from the underlying basement membrane (d) and coincided with tumor cell death in the perivascular cuff (e and $\mathbf{f}$ ). A large majority of the perivascular cuffs in the combination treatment group correlated with changes seen in $\mathbf{e}$ and $\mathbf{f}$. Horizontal bar $=100 \mu \mathrm{m}$.

randomized into 4 groups and treated systemically with either saline control, DC101, vinblastine, or combination therapy for 10 days. FITC-dextran was injected into the lateral tail vein and equilibrated throughout the vascular compartment. The majority of the blood-borne dextran, because of its $148-\mathrm{kDa}$ size, remains intravascular, and despite some perivascular losses due to changes in vascular permeability and the possibility of interstitial hemorrhages, the fluorescence is reflective of the overall volume of blood passing through the tumor vasculature. Because our therapy is chronic in its nature, changes in intratumoral vascular/blood volume are likely to represent structural changes rather than transient fluxes in vascular permeability. By these criteria, DC101 alone caused a $47 \%$ decrease in tumor perfusion, vinblastine alone resulted in a $41 \%$ decrease, and the combination of the 2 drugs resulted in $65 \%$ perfusion inhibition (Figure 5a). Of interest is the appreciable difference in gross vascularity in the corresponding tumor specimens (Figure $5 b$ ).

Effects of chemotherapy treatments on in vivo angiogenesis. The direct assessment of tumor vasculature does not provide any clues as to whether the apparent vascular inhibition within the tumor is primary (cause) or secondary (consequence) to the tumor regression. Evidence for the former would provide support for the hypothesis that low-dose vinblastine treatment alone is potentially antiangiogenic and that the extent of this antiangiogenic effect may be further enhanced by concurrent treatment with DC101. Again, the ratio between intra- and extravascular volume within the tumor could be also somewhat affected by transient changes in vascular permeability (34). To address these questions, we repeated the same fluorescence measurement using an in vivo Matrigel plug angiogenesis assay (ref. 32; personal communication, J. Ahern, Merck $\&$ Co., West Point, Pennsylvania, USA). Four treatment groups were treated with an identical therapeutic regimen as in the tumor perfusion experiment, and the regression of vascularity in subcutaneously implanted Matrigel pellets was quantitatively assessed by measuring the fluorescence of circulating FITC-labeled dextran (Figure 6). DC101 inhibited the bFGF-induced vascularization to $50 \%$ and vinblastine alone to $62.5 \%$ of the positive control. There was again an enhanced effect of the combination therapy, which reduced the Matrigel pellet fluorescence down to $29.2 \%$ of control, a level only marginally different from the negative control (Matrigel not supplemented with growth factors).

\section{Discussion}

Interest in the therapeutic effects of combining antiangiogenic drugs with conventional chemotherapy was initially stimulated by reports from Teicher's laboratory (35) 
showing that the antitumor effects of MTDs of various chemotherapeutic cytotoxic drugs can be augmented, at least in preclinical experiments, when they are combined with angiogenesis inhibitors (36). Counterintuitively, such effects have been attributed to greater delivery of the cytotoxic drugs into tumor masses (36). Increasing the efficacy of cyclic high-dose chemotherapy in such a manner, however, would unlikely alter 2 of the major problems associated with the use of chemotherapy given in this way: induction of moderate to severe side effects (e.g., myelosuppression, nausea, and hair loss), and the devel-
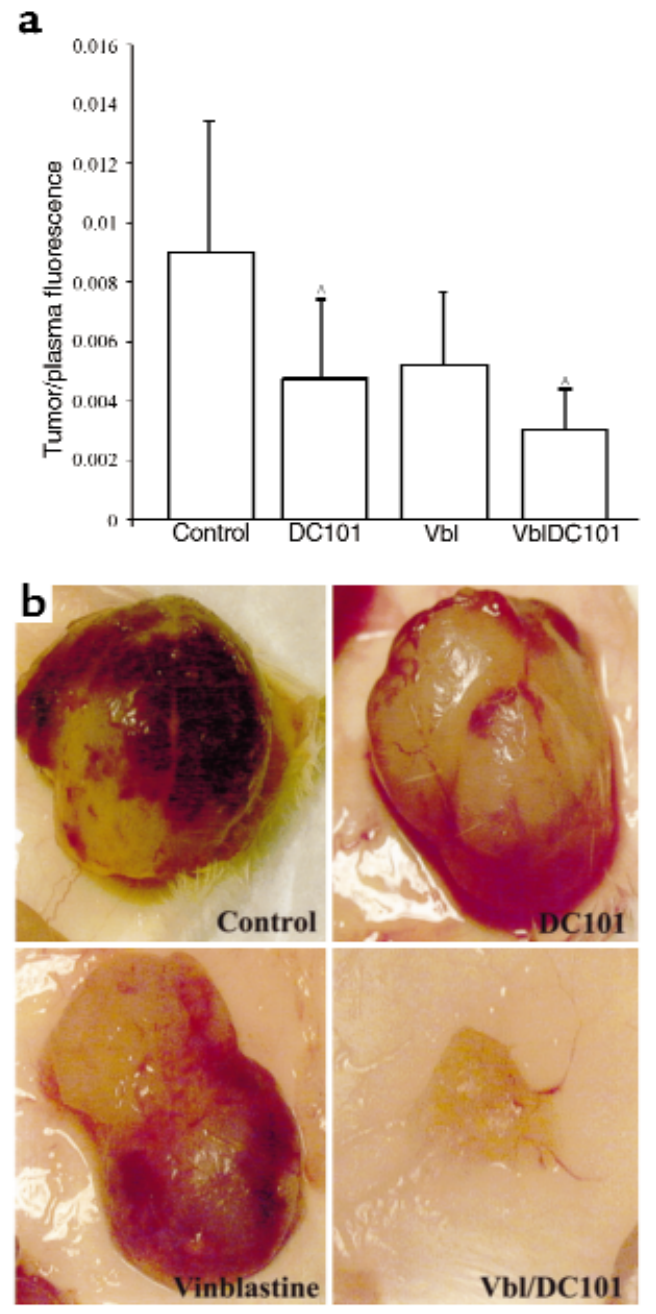

\section{Figure 5}

Impact of antiangiogenic treatment regimen (VbI+DC101) on integrity of tumor vasculature. (a) The decrease in intravascular FITC-dextran fluorescence reflects changes in tumor perfusion in established SK-N-AS neuroblastoma xenografts subjected to a 2-week course of treatment with anti-flk-1 (DC101) antibody, low-dose vinblastine, or combination of the 2 . Both single-drug treatments caused a significant decrease in tumor perfusion, and this effect was enhanced by the combination therapy. Averages of 5 animals (bearing bilateral tumor xenografts) and their respective SDs are shown $\left({ }^{*} P=0.05\right)$. (b) Tumor appearance in treated and untreated animals at the time of excision. Notable is the change in tumor vascularity in the singletreatment groups even before an appreciable change in tumor size. Groups were treated for 14 days before specimen collection. opment of acquired resistance to the cytotoxic drugs. In contrast, our results suggest that these problems can be significantly attenuated, and perhaps even avoided, by using much more frequent administration of significantly lower doses of a chemotherapeutic drug, e.g., vinblastine, when given in combination with anti-VEGF receptor-2 neutralizing antibodies, without sacrificing potent antitumor efficacy. Thus, large $\left(0.75 \mathrm{~cm}^{3}\right)$ established human neuroblastoma xenografts in SCID mice could be induced to regress completely with this combination strategy, whereas either agent alone caused only partial and temporary regressions, with relapses observed in all treated animals 30-50 days after initiation of the individual therapy treatments. In striking contrast, a fully regressed state could be induced and maintained for as long as the combination therapy was maintained, which in our case was 210 days, in the absence of any significant toxicity, as assessed by lack of weight loss. No myelosuppression has been observed either (Klement et al., unpublished observations).

The dose of vinblastine used in our experiments was in the range of $1.5 \mathrm{mg} / \mathrm{m}^{2}$, every 3 days, which is approximately $1 / 4$ of the MTD of this drug in humans, and $1 / 16-1 / 20$ of the MTD in mice, given the fact that the MTD of vinblastine in mice is 4-5 times higher than in humans $(37,38)$. Similar to Browder et al., who used low-dose cyclophosphamide therapy (26), we found evidence, in our case using the Matrigel plug assay, that continuous low-dose vinblastine administration can cause a direct antiangiogenic effect in vivo.

The decision to use anti-VEGF receptor- 2 antibodies in our combination strategy was based on 3 main considerations. First, dividing endothelial cells in particular may be damaged or killed by exposure to the vinblastine therapy. Second, the extent of this damage may be enhanced in a highly selective manner given the cellular distribution of VEGF receptors, which is mainly, though not exclusively, restricted to activated endothelial cells of newly formed vessels $(2,3,5)$. Third, 1 of the major functions of VEGF is to prevent apoptosis of such endothelial cells during the process of vasculogenesis and angiogenesis $(14,28)$. The prosurvival function of VEGF for activated endothelial cells appears due to its ability to activate PI3 kinase/Akt/PKB (39), and/or upregulate expression of several genes and/or proteins known to have antiapoptotic function such as bcl-2 (40), as well as XIAP (41) and survivin $(41,42)$, the latter 2 being members of the IAP family of apoptosis inhibitors (43). For example, survivin protein expression can be massively upregulated, e.g., 15- to 20-fold (41, 42), and XIAP 4-fold, in human endothelial cells by in vitro by VEGF (41) Moreover, XIAP and survivin (especially XIAP) can confer resistance to chemotherapeutic drugs in tumor cells (44). Thus, blockade of VEGF function could conceivably render surviving endothelial cells more vulnerable to the damaging effects of chemotherapy, especially when the cytotoxic drug is given at low doses, by the possible downregulation of multiple antiapoptotic proteins normally induced by VEGF. A similar reasoning has been 


\section{Figure 6}

Inhibition of angiogenesis in vivo by low-dose vinblastine in combination with anti-flk-1 antibody (DC101). Angiogenesis was induced in subcutaneously implanted Matrigel plugs (Mat) by admixing 500 $\mathrm{ng} / \mathrm{mL}$ of bFGF (Mat+bFGF). The mice were treated with DC101 antibody $(800 \mu \mathrm{g} / \mathrm{mL})$ every 3 days, low-dose vinblastine $\left(1 \mathrm{mg} / \mathrm{m}^{2}\right)$ every 3 days, or combination therapy (Vbl/DC101). After 10 days of treatment, mice were injected intravenously with FITC-dextran; Matrigel plugs were removed; and the volume of new blood vessels was assessed by measurement of intravascular FITC content (normalized to FITC in the circulating plasma). Averages of 5 animals and their respective $\mathrm{SDs}$ are shown $\left({ }^{\mathrm{A} P}=0.05\right)$.

used by others to enhance the collateral damaging effects of ionizing radiation on a tumor's vasculature such that even low doses of radiation can induce detectable antivascular effects in the presence of angiostatin or anti-VEGF antibodies $(13,45)$.

Although our results are consistent with the hypothesis that the antivascular effects of vinblastine are significantly enhanced by combination with anti-VEGF R2 (flk-1) antibodies, other mechanisms may be involved. In this regard, it is important to note that extremely low (e.g., picomolar) doses of vinblastine that are devoid of endothelial cytotoxicity can still block aspects of angiogenesis in vitro or in vivo, using the CAM (chorioallantoic membrane) assay (46). Similarly, low (nanomolar) and noncytotoxic concentrations of camptothecin and topotecan can block endothelial cell functions in vitro that are relevant to angiogenesis (47), and the same appears to be the case for paclitaxel (19). Moreover, the proliferation index of endothelial cells in tumor vessels is usually less than $3 \%$ (48). Thus, other functions such as endothelial cell motility, invasion and vessel remodeling may be blocked or altered by low-dose chemotherapy (46), the magnitude of which might be significantly and selectively enhanced by simultaneous exposure to anti-VEGF receptor-2 antibodies. Suppression of mobilization of bone marrow-derived endothelial cell progenitors to sites of angiogenesis is another possibility $(49,50)$.

The results of Browder et al. and Vacca et al. (26, 46), as well as ours, also raise the important question of what the optimum low dose of a given chemotherapeutic drug is with respect to inducing antiangiogenic effects. An analogous situation has been described recently by Fidler and colleagues with respect to IFN- $\alpha$ as an antiangiogenic agent to treat experimental tumors (51). Whereas very high doses $(70,000 \mathrm{U})$ of IFN- $\alpha$ given on weekly basis had little effect, much lower doses (10,000 U) given on a daily basis strongly inhibited tumor growth by a presumed antiangiogenic mechanism (51). Such results highlight what may be the difficulty in selecting the optimal therapeutic dose of new antiangiogenic drugs for both preclinical studies and clinical trials. This has not been considered to be a problem for conventional antitumor cytotoxic drugs that are generally

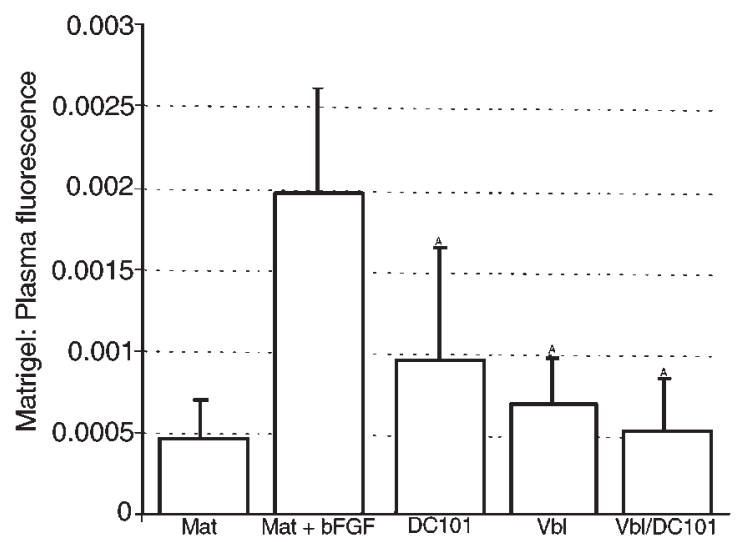

thought to be most effective when used at maximum tolerated doses. However, our results, and those of Browder et al. (26), suggest this may not be the case, provided lower doses are given on a more frequent basis, over long periods, especially in combination with an angiogenesis inhibitor such as anti-VEGF receptor antibodies or TNP-470.

Finally, we would note that there is increasing use of clinical chemotherapy protocols using lower doses of drug given frequently, e.g., weekly, especially as a means of minimizing toxic side effects, such as myelosuppression $(52,53)$, as well as increasing use of oral formulations of chemotherapy (53-55). Such developments make it especially timely to test clinically lowdose regimens of conventional chemotherapeutic drugs alone (53) or in combination with certain newly developed angiogenesis inhibitors, as described here, that can be given continuously, perhaps even on a daily basis, without significant toxic side effects, and which are not rapidly rendered ineffective by development of acquired drug resistance. This prospect also clearly increases the need to begin evaluating in-depth optimal "antivascular" dosing and scheduling characteristics of different chemotherapeutic drugs and to test the effects of such therapeutic approaches in different tumor models. These include orthotopically growing, transplanted tumors, spontaneous tumors arising in transgenic oncomice, as well as models involving treatment of distant metastases, especially of tumors selected for high levels of acquired resistance to the chemotherapeutic drugs used for the low-dose treatment schedules.

\section{Acknowledgments}

This work was inspired by discussions with Dr. J. Folkman. We are grateful for the excellent secretarial assistance of C. Cheng and L. Woodcock. We also greatly appreciate the assistance of J. Huber and A. Santiago in producing the DC101 antibody and P. Huang for her technical assistance. This work was supported by grants to R.S. Kerbel by the CaPCURE Foundation, USA; the National Institutes of Health (CA-41233), and a contract from ImClone Systems. Infrastructural support is also provided by Cancer Care Ontario. G. Klement is a Terry 


\section{Fox Fellow of the National Cancer Institute of Canada.}

1. Folkman, J. 1972. Anti-angiogenesis: new concept for therapy of solid tumors. Ann. Surg. 175:409-416.

2. Kerbel, R.S. 2000. Tumor angiogenesis: past, present, and the near future. Carcinogenesis. 21:505-515.

3. Ferrara, N., and Alitalo, K. 1999. Clinical applications of angiogenic growth factors and their inhibitors. Nat. Med. 5:1359-1364.

4. Presta, L.G., et al. 1997. Humanization of an anti-vascular endothelial growth factor monoclonal antibody for the therapy of solid tumors and other disorders. Cancer Res. 57:4593-4599.

5. Witte, L., et al. 1998. Monoclonal antibodies targeting the VEGF receptor-2 (Flk1/KDR) as an anti-angiogenic therapeutic strategy. Cancer Metastasis Rev. 17:155-161.

6. Prewett, M., et al. 1999. Antivascular endothelial growth factor receptor (fetal liver kinase 1) monoclonal antibody inhibits tumor angiogenesis and growth of several mouse and human tumors. Cancer Res. 59:5209-5218.

7. Fong, T.A., et al. 1999. SU5416 is a potent and selective inhibitor of the vascular endothelial growth factor receptor (Flk-1/KDR) that inhibits tyrosine kinase catalysis, tumor vascularization, and growth of multiple tumor types. Cancer Res. 59:99-106.

8. Lin, P., et al. 1997. Inhibition of tumor angiogenesis using a soluble receptor establishes a role for Tie2 in pathologic vascular growth. J. Clin. Invest. 100:2072-2078

9. Boehm, T., Folkman, J., Browder, T., and O'Reilly, M.S. 1997. Antiangiogenic therapy of experimental cancer does not induce acquired drug resistance. Nature. 390:404-407.

10. Kaban, L.B., et al. 1999. Antiangiogenic therapy of a recurrent giant cell tumor of the mandible with interferon Alfa-2a. Pediatrics. 103:1145-1149.

11. Kerbel, R.S. 1991. Inhibition of tumor angiogenesis as a strategy to circumvent acquired resistance to anti-cancer therapeutic agents. BioEssays. 13:31-36.

12. Kerbel, R.S. 2000. Acquired drug resistance driven by tumor cell genetic instability: circumvention by direct acting anti-angiogenic vascular targeting agents. In DNA alterations in cancer: genetic and epigenetic changes. M. Ehrlich, editor. Biotechniques Books. Natick, MA. 489-501.

13. Gorski, D.H., et al. 1999. Blockage of the vascular endothelial growth factor stress response increases the antitumor effects of ionizing radiation. Cancer Res. 59:3374-3378.

14. Jain, R.K., et al. 1998. Endothelial cell death, angiogenesis, and microvascular function after castration in an androgen-dependent tumor: role of vascular endothelial growth factor. Proc. Natl. Acad. Sci. USA. 95:10820-10825.

15. Benjamin, L.E., Golijanin, D., Itin, A., Pode, D., and Keshet, E. 1999. Selective ablation of immature blood vessels in established human tumors follows vascular endothelial growth factor withdrawal. J. Clin. Invest. 103:159-165.

16. Denekamp, J. 1993. Angiogenesis, neovascular proliferation and vascular pathophysiology as targets for cancer therapy. Br. J. Radiol. 66:181-196.

17. Tannock, I.F. 1970. Population kinetics of carcinoma cells, capillary endothelial cells, and fibroblasts in a transplanted mouse mammary tumor Cancer Res. 30:2470-2476.

18. Tannock, I.F., and Hayashi, S. 1972. The proliferation of capillary endothelial cells. Cancer Res. 32:77-82.

19. Belotti, D., et al. 1996. The microtubule-affecting drug paclitaxel has antiangiogenic activity. Clin. Cancer Res. 2:1843-1849.

20. O'Leary, J.J., et al. 1999. Antiangiogenic effects of camptothecin analogues 9-amino-20(S)-camptothecin, topotecan, and CPT-11 studied in the mouse cornea model. Clin. Cancer Res. 5:181-187.

21. Baguley, B.C., Holdaway, K.M., Thomsen, L.L., Zhuang, L., and Zwi, L.J. 1991. Inhibition of growth of colon 38 adenocarcinoma by vinblastine and colchicine: evidence for a vascular mechanism. Eur. J. Cancer. 27:482-487.

22. Schirner, M., Hoffmann, J., Menrad, A., and Schneider, M.R. 1998. Antiangiogenic chemotherapeutic agents: characterization in comparison to their tumor growth inhibition in human renal cell carcinoma models. Clin. Cancer Res. 4:1331-1336

23. Presta, M., et al. 1999. Purine analogue 6-methylmercaptopurine riboside inhibits early and late phases of the angiogenesis process. Cancer Res. 59:2417-2424.

24. Steiner, R. 1992. Angiostatic activity of anticancer agents in the chick embryo chorioallantoic membrane (CHE-CAM) assay. In Angiogenesis: key principles-science-technology-medicine. R. Steiner, P.S. Weiss, and R. Langer, editors. Birkhauser Verlag. Basel, Switzerland. 449-454.

25. Yoshikawa, A., Saura, R., Matsubara, T., and Mizuno, K. 1997. A mechanism of cisplatin action: antineoplastic effect through inhibition of neovascularization. Kobe. J. Med. Sci. 43:109-120.

26. Browder, T., et al. 2000. Antiangiogenic scheduling of chemotherapy improves efficacy against experimental drug-resistant cancer. Cancer Res. In press.

27. Hirata, S., Matsubara, T., Saura, R., Tateishi, H., and Hirohata, K. 1989. Inhibition of in vitro vascular endothelial cell proliferation and in vivo neovascularization by low-dose methotrexate. Arthritis Rheum. 32:1065-1073.
28. Alon, T., et al. 1995. Vascular endothelial growth factor acts as a survival factor for newly formed retinal vessels and has implications for retinopathy of prematurity. Nat. Med. 1:1024-1028.

29. Yuan, F., et al. 1996. Time-dependent vascular regression and permeability changes in established human tumor xenografts induced by an anti-vascular endothelial growth factor/vascular permeability factor antibody. Proc. Natl. Acad. Sci. USA. 93:14765-14770.

30. Stram, D.O., et al. 1996. Consolidation chemoradiotherapy and autologous bone marrow transplantation versus continued chemotherapy for metastatic neuroblastoma: a report of two concurrent Children's Cancer Group studies. J. Clin. Oncol. 14:2417-2426.

31. Rockwell, P., Neufeld, G., Glassman, A., Caron, D., and Goldstein, N. 1995. In vitro neutralization of vascular endothelial growth factor activation of flk-1 by a monoclonal antibody. Mol. Cell. Different. 3:91-109.

32. Passaniti, A., et al. 1992. A simple, quantitative method for assessing angiogenesis and antiangiogenic agents using reconstituted basement membrane, heparin, and fibroblast growth factor. Lab. Invest. 67:519-528.

33. Bordow, S.B., et al. 1994. Expression of the multidrug resistance-associated protein (MRP) gene correlates with amplification and overexpression of the $\mathrm{N}$-myc oncogene in childhood neuroblastoma. Cancer Res. 54:5036-5040.

34. Jain, R.K. 1990. Vascular and interstitial barriers to delivery of therapeutic agents in tumors. Cancer Metastasis Rev. 9:253-266.

35. Teicher, B.A., Sotomayor, E.A., and Huang, Z.D. 1992. Antiangiogenic agents potentiate cytotoxic cancer therapies against primary and metastatic disease. Cancer Res. 52:6702-6704.

36. Kakeji, Y., and Teicher, B.A. 1997. Preclinical studies of the combination of angiogenic inhibitors with cytotoxic agents. Invest. New Drugs. 15:39-48.

37. Tashiro, T., et al. 1989. Responsiveness of human lung cancer/nude mouse to antitumor agents in a model using clinically equivalent doses. Cancer Chemother. Pharmacol. 24:187-192.

38. Inaba, M., et al. 1989. Evaluation of antitumor activity in a human breast tumor/nude mouse model with a special emphasis on treatment dose. Cancer. 64:1577-1582.

39. Gerber, H.P., et al. 1998. Vascular endothelial growth factor regulates endothelial cell survival through the phosphatidylinositol 3'-kinase/Akt signal transduction pathway. Requirement for Flk-1/KDR activation. J. Biol. Chem. 273:30336-30343.

40. Nor, J.E., Christensen, J., Mooney, D.J., and Polverini, P.J. 1999. Vascular endothelial growth factor (VEGF)-mediated angiogenesis is associated with enhanced endothelial cell survival and induction of Bcl-2 expression. Am.J. Pathol. 154:375-381.

41. Tran, J., et al. 1999. Marked induction of the IAP family anti-apoptotic proteins survivin and XIAP by VEGF in vascular endothelial cells. Biochem. Biophys. Res. Commun. 264:781-788.

42. O'Connor, D.S., et al. 2000. Control of apoptosis during angiogenesis by survivin expression in endothelial cells. Am. J. Pathol. 156:393-398.

43. LaCasse, E.C., Baird, S., Korneluk, R.G., and MacKenzie, A.E. 1998. The inhibitors of apoptosis (IAPs) and their emerging role in cancer. Oncogene. 17:3247-3259.

44. Tamm, I., et al. 1998. IAP-family protein survivin inhibits caspase activity and apoptosis induced by Fas (CD95), Bax, caspases, and anticancer drugs. Cancer Res. 58:5315-5320.

45. Mauceri, H.J., et al. 1998. Combined effects of angiostatin and ionizing radiation in antitumour therapy. Nature. 394:287-291.

46. Vacca, A., et al. 1999. Antiangiogenesis is produced by nontoxic doses of vinblastine. Blood. 94:4143-4155.

47. Clements, M.K., Jones, C.B., Cumming, M., and Daoud, S.S. 1999. Antiangiogenic potential of camptothecin and topotecan. Cancer Chemother. Pharmacol. 44:411-416.

48. Vartanian, R.K., and Weidner, N. 1995. Endothelial cell proliferation in prostatic carcinoma and prostatic hyperplasia: correlation with Gleason's score, microvessel density, and epithelial cell proliferation. Lab. Invest. 73:844-850.

49. Asahara, T., et al. 1999. VEGF contributes to postnatal neovascularization by mobilizing bone marrow-derived endothelial progenitor cells. EMBOJ. 18:3964-3972

50. Ito, H., et al. 1999. Endothelial progenitor cells as putative targets for angiostatin. Cancer Res. 59:5875-5877.

51. Slaton, J.W., Perrotte, P., Inoue, K., Dinney, C.P., and Fidler, I.J. 1999. Interferon-alpha-mediated down-regulation of angiogenesis-related genes and therapy of bladder cancer are dependent on optimization of biological dose and schedule. Clin. Cancer Res. 5:2726-2734.

52. Hainsworth, J.D., Burris, H.A., Erland, J.B., Thomas, M., and Greco, F.A. 1998. Phase I trial of docetaxel administered by weekly infusion in patients with advanced refractory cancer.J.Clin. Oncol. 16:2164-2168.

53. Rocca, A., et al. 1999. Low dose oral Methotrexate (MTX) and Cyclophosphamide (CTX) in metastatic breast cancer (MBC): antitumor activity and correlation with serum vascular endothelial growth factor (VEGF) levels. Proc. Am. Soc. Clin. Oncol. 18:121a. (Abstr.)

54. McLeod, H.L., and Evans, W.E. 1999. Oral cancer chemotherapy: the promise and the pitfalls. Clin. Cancer Res. 5:2669-2671.

55. Lamont, E.B., and Schilsky, R.L. 1999. The oral fluoropyrimidines in cancer chemotherapy. Clin. Cancer Res. 5:2289-2296. 